DOI: http://doi.org/10.4038/sljid.v7i0.8201

\title{
Osteoarticular melioidosis: A bone to pick about an emerging but neglected tropical disease
}

\author{
SReddy, TShaw, TAKChaitanya, KEVandana, AKBhat, CMukhopadhyay
}

\section{Introduction}

Melioidosis, caused by the soil inhabitant Burkholderiapseudomallei, often presents with osteoarticular manifestations in endemic settings, where the mode of infection is through inoculation. Bacteraemic forms of the disease, which is associated with high case fatality rates, are often due to the systemic dissemination of bacteria from osteoarticular infections. With increasing evidence suggesting the endemicity of melioidosis in India, the present study was undertaken to elucidate the clinical, microbiological and epidemiological characteristics of patients diagnosed with osteoarticular forms of melioidosis.

\section{Methods}

Data obtained from 200 patients diagnosed with melioidosis (culture-confirmed) from January 2006 to December 2016 at a tertiary care teaching hospital in South-West India was used in the present study. Baseline demographic and clinical characteristics of patients with osteoarticular form of melioidosis among the study cohort were further analyzed.

\section{Results}

Osteoarticular melioidosis accounted for $17.5 \%$ (35/200) of all cases of melioidosis in the given period, comprising of $29(82.8 \%)$ males and $6(17.1 \%)$ females. Mean age of the study population was $47.7 \pm 13.6$ (Range 7-67). Twenty-four cases (68.5\%) presented with monoarticular septic arthritis, while $11(31.4 \%)$ had osteomyelitis. The most commonly affected site was the knee joint $(25,71.4 \%)$, followed by vertebrae $(3 ; 1$ thoracic and 2 lumbar) and ankle joint. Diabetes mellitus was the most common co-morbid condition $(29,82.9 \%)$, followed by chronic alcoholism $(9,25.7 \%)$ and chronic kidney disease $(6,17.1 \%)$. Bacteremia was documented in $15(42.8 \%)$ cases. Case fatality was $8.5 \%(n=3)$.

\section{Discussion \& Conclusion}

Early diagnosis and befitting management of osteoarticular melioidosis can prevent systemic dissemination of the bacteria and thus reduce the associated case-fatality rates. Microbiological culture results play an indispensable role in diagnosis of osteoarticular melioidosis.

Departments of Microbiology and Orthopedics, Kasturba Medical College and Hospital, Manipal, India Address for correspondence: Prof C Mukhopadhyay, Department of Microbiology, Kasturba Medical College,

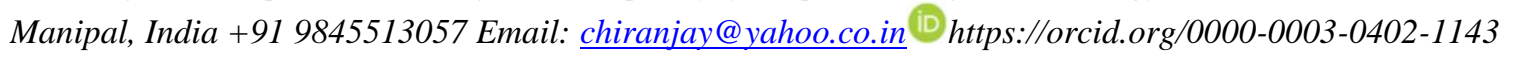

